\title{
Management consideration for patient with a large solitary fibrous tumor occupying the infratemporal fossa: A case report
}

\author{
MAKOTO ADACHI ${ }^{1,2}$, MASAYUKI MOTOHASHI ${ }^{1}$, YASUHIKO KAKU ${ }^{3}$, SHIGEYUKI SUGIE ${ }^{4}$, \\ YASUNORI MURAMATSU ${ }^{1}$ and SHINICHIRO SUMITOMO ${ }^{2}$
}

\author{
${ }^{1}$ Department of Oral and Maxillofacial Surgery, Asahi University Murakami Memorial Hospital, Gifu 500-8523; \\ ${ }^{2}$ Department of Oral and Maxillofacial Surgery, Division of Oral Pathogenesis and Disease Control, Asahi University, \\ School of Dentistry, Mizuho, Gifu 501-0296; ${ }^{3}$ Department of Neurosurgery, Asahi University Murakami Memorial Hospital, \\ Gifu 500-8523; ${ }^{4}$ Department of Pathology, Asahi University Murakami Memorial Hospital, Gifu 500-8523, Japan
}

Received November 9, 2017; Accepted December 22, 2017

DOI: $10.3892 / \mathrm{mco} .2018 .1575$

\begin{abstract}
The solitary fibrous tumor (SFT) was first described as a mesenchymal tumor of fibroblastic type, present in the pleura. The head and neck area is the third most common site of SFT occurrence. Numb chin syndrome (NCS) is a rare neurological symptom. The current report describes the case of a 39-year-old female patient referred to the present hospital with NCS, which was later observed to be due to a large SFT in the infratemporal fossa. Computed tomography and magnetic resonance images were taken and subsequent examination of these images revealed a space occupying mass $(52 \times 50 \times 40 \mathrm{~mm})$ in the infratemporal fossa. An open biopsy was performed on the lower part of the right auricula following angiography assisted vascular embolization of the maxillary artery. Pathological diagnosis was verified as SFT. Despite the tumor size and anatomical site, the SFT was successfully reduced in size by treatment with intensity modulated radiation therapy (IMRT) alone, with no recurrence. IMRT treatment of SFT may be effective as a future potential option for locally advanced head and neck SFT.
\end{abstract}

\section{Introduction}

Solitary fibrous tumors (SFT), a rare mesenchymal neoplasm originally described in the pleura by Klempere and Rabin in 1931 (1), are uncommon spindle cell neoplasms of mesenchymal origin, but more recently reported at a wide range of anatomic sites.

Correspondence to: Dr Makoto Adachi, Department of Oral and Maxillofacial Surgery, Division of Oral Pathogenesis and Disease Control, Asahi University, School of Dentistry, 1851 Hozumi, Mizuho, Gifu 501-0296, Japan

E-mail: madachi_dds_phd@yahoo.co.jp

Key words: solitary fibrous tumor, numb chin syndrome, intensity modulated radiation therapy, infratemporal fossa, vascular tumor
Hemangiopericytoma (HPC) is a very rare vascular tumor and originates from small pericapillary spindle cells called Zimmermann's pericytes, which was first described by Stout and Murray in 1942 as a tumor of the retroperitoneum, buttock, and thigh (2). HPC has been considered as a cellular variant of SFT. In the 2013 World Health Organization (WHO) classification of tumors of the soft tissue, HPC was removed as a synonym for SFT (3).

The predilection sites of this tumor are the lower limbs, axilla, and pelvis as well as the head and neck (4). The head and neck area is the third most common site, accounting for $15-30 \%$ of all occurrences. In the head and neck region, the tumor usually involves the orbit, nasal cavity, oral cavity, jaw, parotid gland, parapharyngeal space or jugular foramen (5).

Numb chin syndrome (NCS) is a rare neurological symptom and an indicator of various diseases. It describes a condition presenting as orofacial sensory disturbance especially localized to the lower lip and chin. The condition manifests spontaneously without history of trauma, infection or obvious dental cause. NCS is an important finding as it may be the initial symptom of Multiple Sclerosis or metastatic malignancy (6).

In this paper, we report a case of a large SFT in the infratemporal fossa which manifested NCS as the initial symptom, and was treated with intensity modulated radiation therapy alone.

\section{Case report}

An otherwise healthy 39-year-old female patient was referred to our hospital having experienced right lower lip numbness for one month. She had no past medical history. Initial findings indicated no facial swelling or trismus. Intra-oral findings revealed no abnormal observation. Panoramic radiography showed that there were no bony lesions at the mandible. However, the right lower wisdom tooth showed signs of pericoronitis (Fig. 1). The patient received medication with antibiotics and Vitamin B12 to improve the pericoronitis and right lower lip numbness. Two weeks following referral, in spite of these medications, right lower lip numbness was not improved. Three months later, the right lower wisdom tooth was extracted, and subsequently right lower lip numbness was slightly improved. 
At five months post referral the lower part of the right auricula displayed swelling. Computed tomography (CT) and magnetic resonance imaging (MRI) revealed a space occupying mass $(52 \times 50 \times 40 \mathrm{~mm})$ in the infratemporal fossa (Figs. 2 and 3). Fine needle aspiration biopsy (FNAB) was performed at this site and hemoid gelatinous fluid was withdrawn. However, pathological examination of the sample did not lead to final diagnosis. Parotid gland tumor, neurilemoma, primitive neuroectodermal tumor (PNET), conventional HPC, and SFT were cited as differential diagnoses.

An open biopsy was performed on the lower part of the right auricula following angiography assisted vascular embolization of the maxillary artery (Fig. 4A and B). The tumor was found in deeper layers than the parotid gland, and bleeding was not observed during biopsy due to the effect of vascular embolization (Fig. 4C). Immunohistologically, CD99 was positive, CD34 was positive only for vascular endothelium, and B cell lymphoma 2 (Bcl-2) was weakly positive. These findings suggested SFT. Then, athological diagnosis was confirmed as SFT (Fig. 5A-H).

We offered the patient intensity modulated radiation therapy (IMRT) and obtained written informed consent. IMRT of the primary lesion was performed five times per week at two Gray (Gy) per fraction, for a total of $66 \mathrm{~Gy}$. The treatment was continued, with radiation being administered for about six weeks. The patient developed grade two stomatitis, dry mouth, grade two dermatitis, grade two alopecia, and grade two dysgeusia (National Cancer Institute common toxicity criteria, version 4.0).

Successful shrinkage of the tumor was seen in MRI 3 years after treatment without recurrence (Fig. 6). Slight numbness of the lower lip, trismus, occasional light dizziness and sustained tinnitus remained as problematic disorders.

\section{Discussion}

SFT originating in the infratemporal fossa was first described by Buchanan (7), followed by several reports showing SFT arising from the infra temporal fossa (8-10). These case reports showed swelling in the temporal region without pain, and they did not show nerve palsy as a first manifestation. In the case presented, the patient with a huge SFT at the infratemporal fossa displayed numb chin syndrome as a first manifestation and the period from first presentation to final diagnosis took approximately 6 months. NCS is mostly caused by odontogenic lesions, commonly recognized as symptoms of Vincent's disease. NCS can also be caused by malignant tumor, infectious disease, degenerative disease or metabolic disease in an area supplied by the submandibular nerve (6). NCS should be differentially diagnosed not only for odontogenic diseases but also the possibility of various other causes, and sufficient image examination should be considered.

SFT are a heterogeneous group of rare spindle-cell tumors including benign and malignant neoplasms (11). In this case, NCS may be due to the expansion of the tumor and compression of the trigeminal nerve third branch.

SFT originate from mesothelial mesenchymal cells, whereas HPC originates from capillary pericytes. These tumors have much in common in clinical behavior, pathological findings and image findings. HPC is now considered

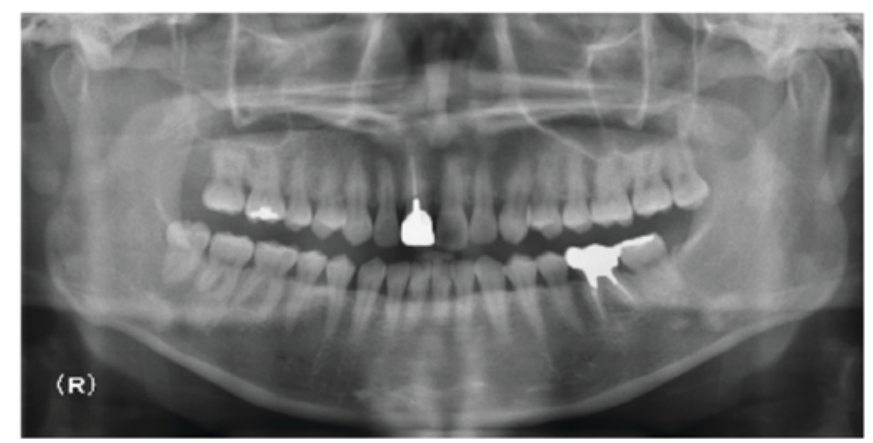

Figure 1. Panoramic radiograph. There were no bony lesions at the mandible.
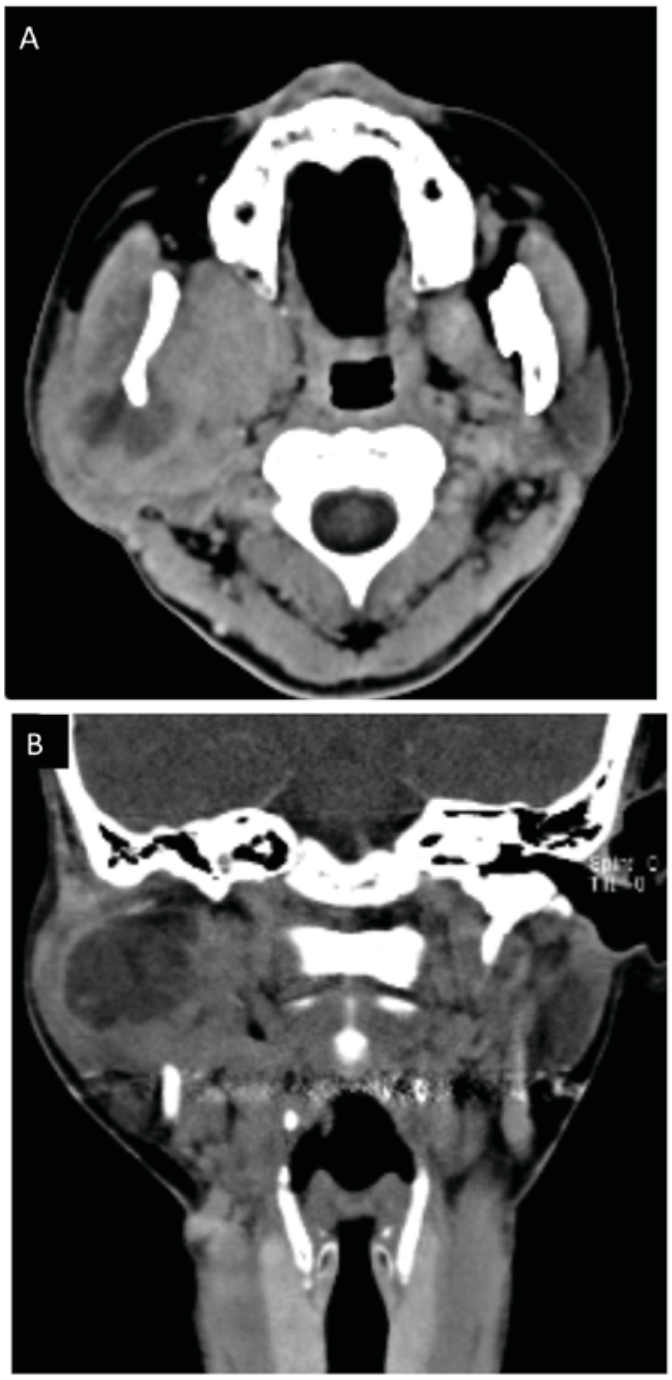

Figure 2. Computed tomography (CT) before treatment. (A) Horizontal and (B) coronal.

to be a cellular variant of SFT. The 2006 WHO fascicle of soft tissue tumors stated the histological appearance and clinical behavior of HPC and SFT are similar, a view also shared by others (12). In the 2013 WHO classification of soft tissue tumors, the terminology is unified as SFT only (13). In the case presented, the tumor was diagnosed as HPC by 

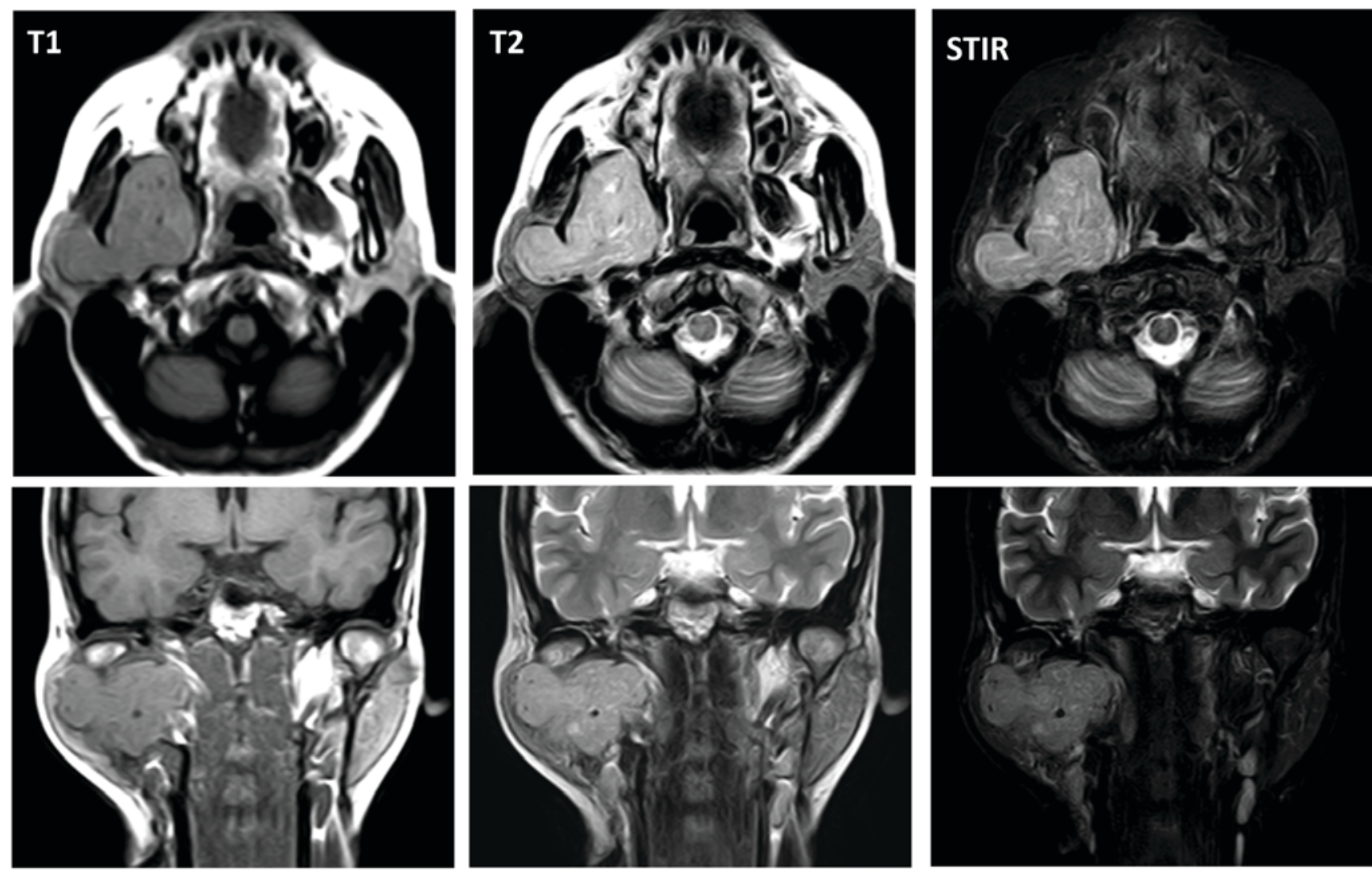

Figure 3. Magnetic resonance imaging (MRI) before treatment.
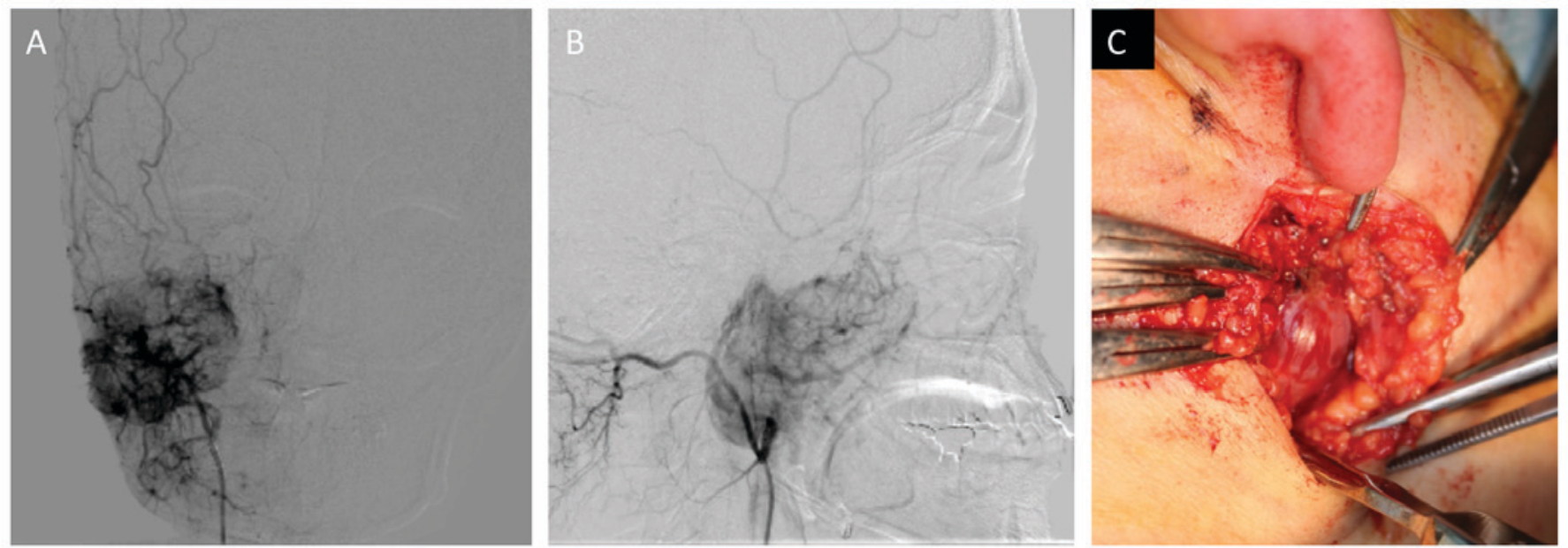

Figure 4. (A) Frontal view of angiography, (B) Side view of angiography, (C) intra-operating view of open biopsy.

histopathological examination including immunostaining, but it was difficult to distinguish from SFT using image findings alone.

The standard treatment of SFT is still controversial. The optimal treatment for SFT is considered to be radical resection of the tumor (14). SFT is considered to be a radiosensitive tumor (10). The combination of radical surgery and post-operative radiation therapy was suggested to reduce the rate of local recurrence (15). Cytotoxic chemotherapy such as doxorubicin-based, gemcitabine-based, and paclitaxel-based are used for patients with locally advanced, recurrent, or metastatic SFT, and show a poor response (16). Recent novel targeted agents such as temozolomide-bevacizumab combination therapy (17), sunitinib (18), sorafenib (19), pazopanib (20) and anti-insulin-like growth factor I agents (21) showed more favorable results than conventional cytotoxic chemotherapy in advanced SFT patients. In our case, we opted for a single treatment by IMRT to the SFT at the infratemporal fossa because surgical access may be difficult as this area includes the lower cranial nerve, internal carotid artery, and internal jugular vein.

Despite the tumor size and anatomical site, tumor shrinkage was obtained successfully without relapse/recurrence. IMRT 


\section{A}
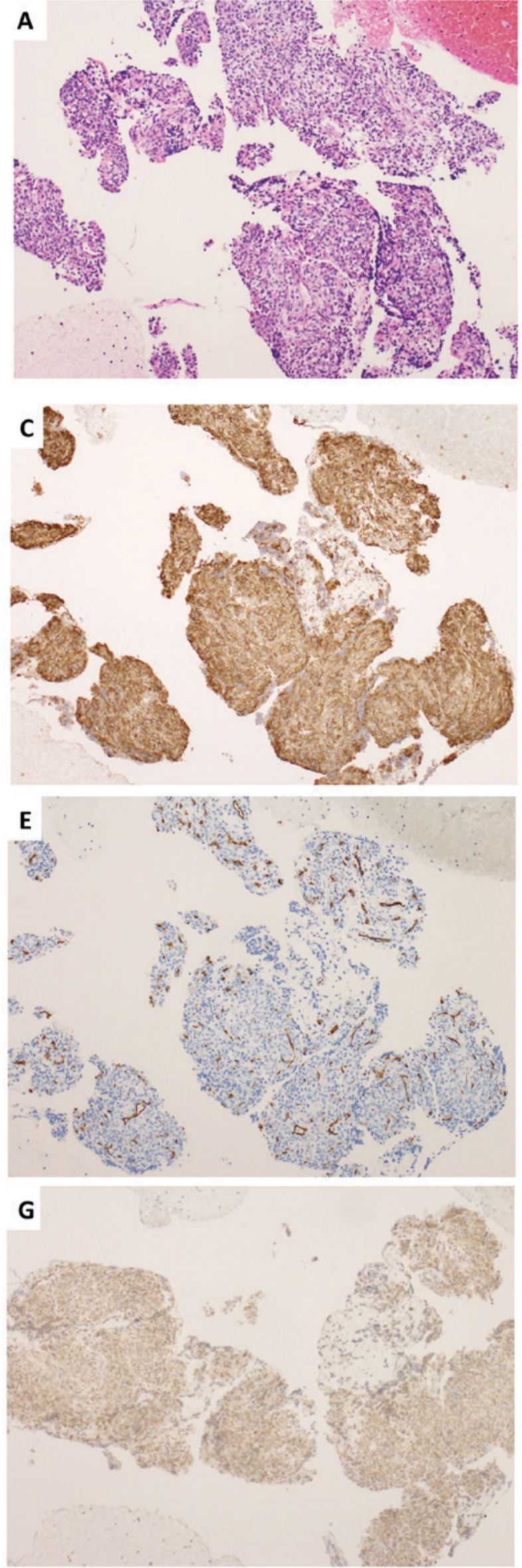
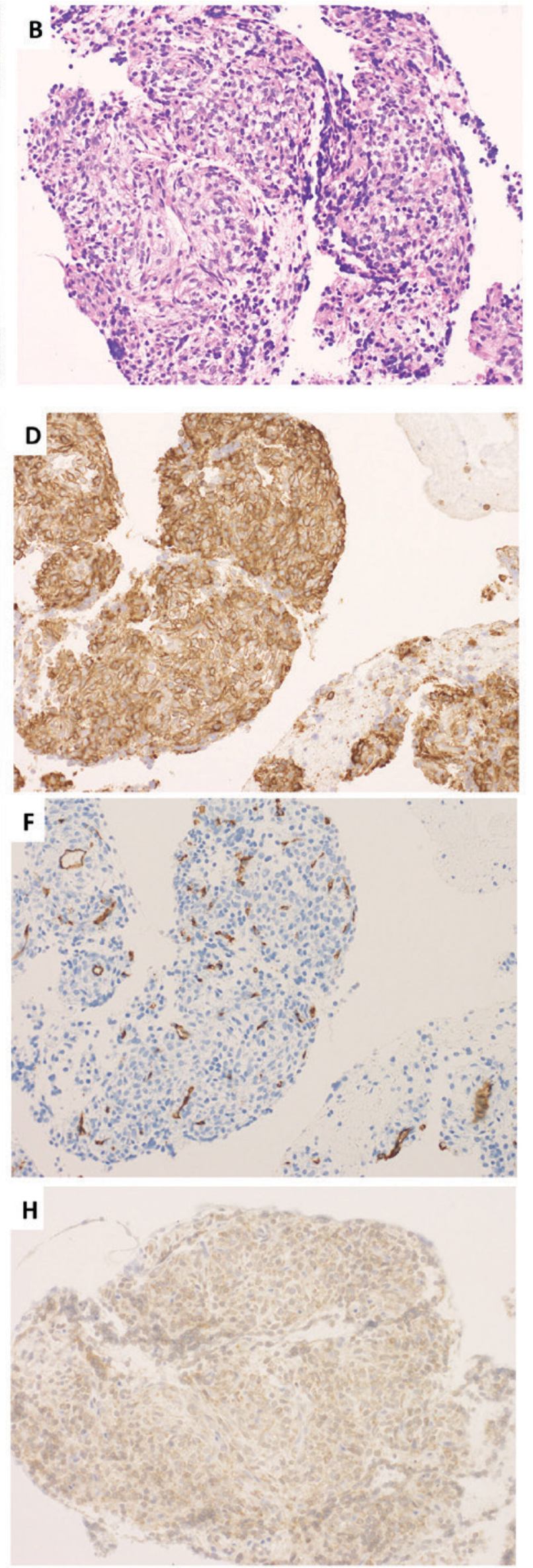

Figure 5. Pathological findings. (A) Hematoxylin and eosin staining (original magnification, x10), (B) Hematoxylin and eosin staining (original magnification, x20). (C) CD99 staining (original magnification, x10), (D) CD99 staining (original magnification, x20), (E) CD34 staining (original magnification, x10), (F) CD34 staining (original magnification, x20), (G) Bcl-2 staining (original magnification, x10), (H) Bcl-2 staining (original magnification, x20). 

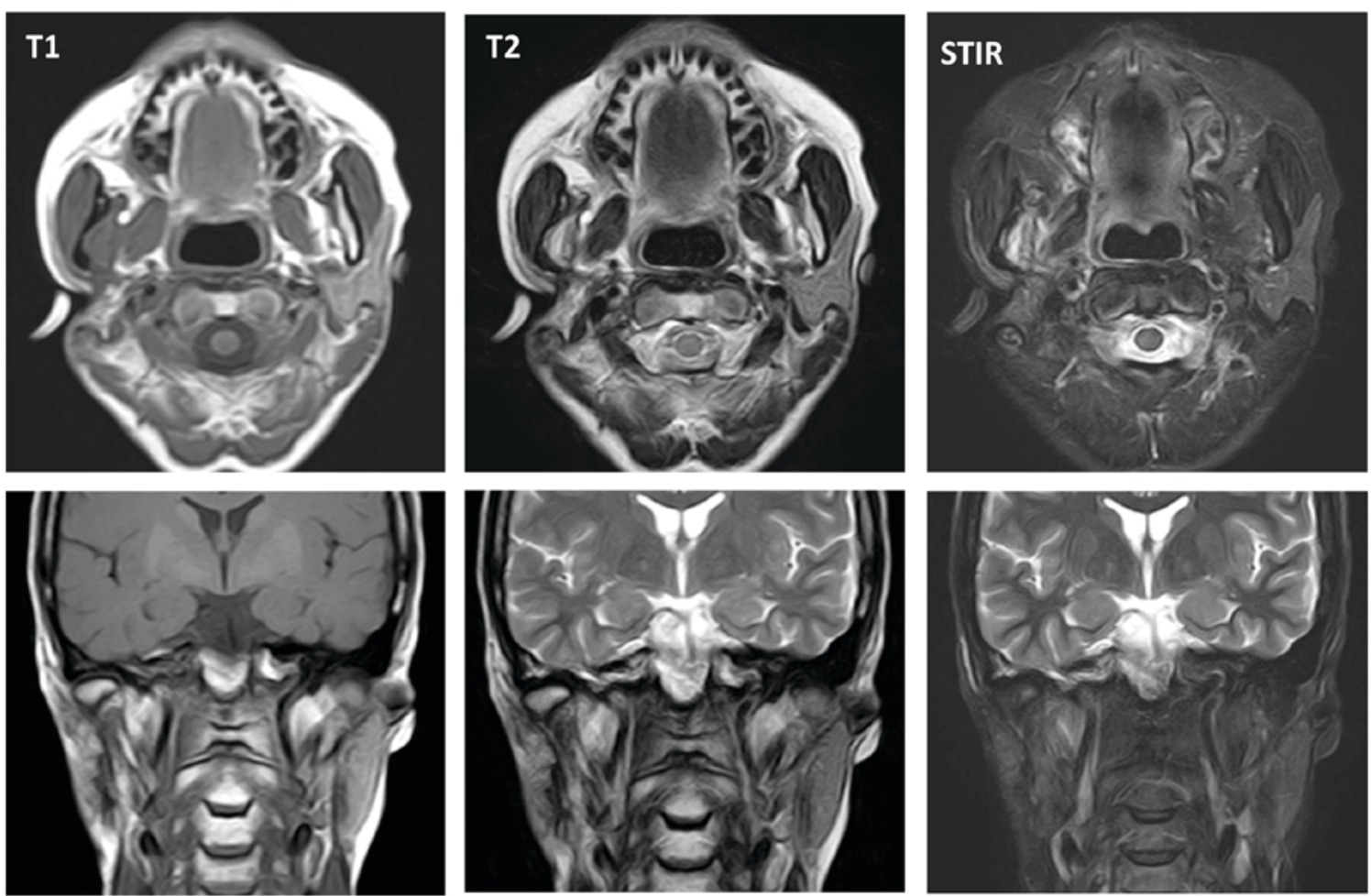

Figure 6. Magnetic resonance imaging (MRI) three years after treatment.

alone may be effective as a future potential treatment option for locally advanced head and neck SFT.

\section{References}

1. Klemperer P and Rabin C: Primary neoplasms of the pleura. A report of five cases. Am J Ind Med 22: 1-31, 1992.

2. Stout AP and Murray MR: Hemangiopericytoma: A vascular tumor featuring zimmermann's pericytes. Ann Surg 116: 26-33, 1942.

3. Doyle LA: Sarcoma classification: An update based on the 2013 World Health Organization Classification of Tumors of Soft Tissue and Bone. Cancer 120: 1763-1774, 2014.

4. Koch M, Nielsen GP and Yoon SS: Malignant tumors of blood vessels: Angiosarcomas, hemangioendotheliomas, and hemangioperictyomas. J Surg Oncol 97: 321-329, 2008.

5. Fareed MM, Al Amro AS, Akasha R, Al Assiry M, Al Asiri M, Tonio $\mathrm{M}$ and Bayoumi Y: Parapharyngeal space hemangiopericytoma treated with surgery and postoperative radiation-a case report. Head Neck Oncol 4: 10, 2012.

6. Smith RM, Hassan A and Robertson CE: Numb chin syndrome. Curr Pain Headache Rep 19: 44, 2015.

7. Buchanan G: Two rare tumours involving the infratemporal fossa: Alveolar soft part sarcoma and haemangiopericytoma. J Laryngol Otol 89: 375-389, 1975

8. Kanazawa T, Nishino H, Miyata M, Kuriki K, Abe K and Ichimura K: Haemangiopericytoma of infratemporal fossa. J Laryngol Otol 115: 77-79, 2001.

9. Brucoli M, Giarda M, Valente $G$ and Benech A Hemangiopericytoma of the infratemporal fossa: Progression toward malignancy in a 30-year history. J Craniofac Surg 16: $1146-1150,2005$

10. Bianchi B, Poli T, Bertolini F and Sesenna E: Malignant hemangiopericytoma of the infratemporal fossa: Report of a case. J Oral Maxillofac Surg 60: 309-312, 2002.

11. Penel N, Amela EY, Decanter G, Robin YM and Marec-Berard P Solitary fibrous tumors and so-called hemangiopericytoma. Sarcoma 2012: 690251, 2012.

12. Ambrosini-Spaltro A and Eusebi V: Meningeal hemangiopericytomas and hemangiopericytoma/solitary fibrous tumors of extracranial soft tissues: A comparison. Virchows Arch 456: 343-354, 2010.
13. Fletcher CD, Bridge $\mathrm{J}$ and Lee $\mathrm{J}$ : World Health Organization Classification of Tumours of Soft Tissue and Bone. IARC Press, Lyon, France, 2013.

14. Koscielny S, Bräuer B and Förster G: Hemangiopericytoma: A rare head and neck tumor. Eur Arch Otorhinolaryngol 260: 450-453, 2003.

15. Schirmer CM and Heilman CB: Hemangiopericytomas of the skull base. Neurosurg Focus 30: E10, 2011.

16. Park MS, Ravi V, Conley A, Patel SR, Trent JC, Lev DC, Lazar AJ, Wang WL, Benjamin RS and Araujo DM: The role of chemotherapy in advanced solitary fibrous tumors: A retrospective analysis. Clin Sarcoma Res 3: 7, 2013.

17. Park MS, Patel SR, Ludwig JA, Trent JC, Conrad CA, Lazar AJ, Wang WL, Boonsirikamchai $\mathrm{P}$, Choi $\mathrm{H}$, Wang $\mathrm{X}$, et al: Activity of temozolomide and bevacizumab in the treatment of locally advanced, recurrent, and metastatic hemangiopericytoma and malignant solitary fibrous tumor. Cancer 117: 4939-4947, 2011.

18. Stacchiotti S, Negri T, Palassini E, Conca E, Gronchi A, Morosi C, Messina A, Pastorino U, Pierotti MA, Casali PG and Pilotti S: Sunitinib malate and figitumumab in solitary fibrous tumor: Patterns and molecular bases of tumor response. Mol Cancer Ther 9: 1286-1297, 2010.

19. Domont J, Massard C, Lassau N, Armand JP, Le Cesne A and Soria JC: Hemangiopericytoma and antiangiogenic therapy: Clinical benefit of antiangiogenic therapy (sorafenib and sunitinib) in relapsed malignant haemangioperyctoma/solitary fibrous tumour. Invest New Drugs 28: 199-202, 2010.

20. Sleijfer S, Ray-Coquard I, Papai Z, Le Cesne A, Scurr M, Schöffski P, Collin F, Pandite L, Marreaud S, De Brauwer A, et al: Pazopanib, a multikinase angiogenesis inhibitor, in patients with relapsed or refractory advanced soft tissue sarcoma: A phase II study from the European organisation for research and treatment of cancer-soft tissue and bone sarcoma group (EORTC study 62043). J Clin Oncol 27: 3126-3132, 2009.

21. Quek R, Wang Q, Morgan JA, Shapiro GI, Butrynski JE, Ramaiya N, Huftalen T, Jederlinic N, Manola J, Wagner AJ, et al: Combination mTOR and IGF-1R inhibition: Phase I trial of everolimus and figitumumab in patients with advanced sarcomas and other solid tumors. Clin Cancer Res 17: 871-879, 2011. 